\title{
Tipificaciones de los nombres publicados por E. Hassler y R. Chodat en el género Dorstenia L. (Moraceae) de la flora del Paraguay
}

\author{
Lorenzo Ramella, Mónica Soloaga \& Patrick Perret
}

\begin{abstract}
RAMELLA, L., M. SOLOAGA \& P. PERRET (2014). Typifications of names published by E. Hassler and R. Chodat in the genus Dorstenia L. (Moraceae) of the flora of Paraguay. Candollea 69: 207-210. In Spanish, English and Spanish abstracts.

The authors typify 7 names in the genus Dorstenia L. published by Emil Hassler and Robert Chodat based on specimens collected in Paraguay. For each name, the typical material in accordance to the original descriptions and used by these authors is identified. A typographical error in the designation of the holotype of Dorstenia cayapia subf. lobata Hassl. is corrected. The prior lectotypification of Dorstenia cayapia f. paraguariensis Hassl. is narrowed down to a single specimen. Dorstenia brasiliensis var. guaranitica Chodat is a superfluous illegitimate name.
\end{abstract}

\section{Key-words}

MORACEAE - Dorstenia - Typification - Paraguay

\section{Resumen}

RAMELLA, L., M. SOLOAGA \& P. PERRET (2014). Tipificaciones de los nombres publicados por E. Hassler y R. Chodat en el género Dorstenia L. (Moraceae) de la flora del Paraguay. Candollea 69: 207-210. En español, resúmenes en inglés y español.

Los autores efectúan la tipificación de 7 nombres en el género Dorstenia L. publicados por Emil Hassler y Robert Chodat en base a plantas colectadas en Paraguay. Para cada uno de los nombres, se identifica el material típico que corresponde a las descripciones originales y que fue visto por estos autores. Un error tipográfico en la designación del holotipo de Dorstenia cayapia subf. lobata Hassl. es corregido. Se precisa la lectotipificación anterior de Dorstenia cayapia f. paraguariensis Hassl. designando un único espécimen. Dorstenia brasiliensis var. guaranitica Chodat es un nombre superfluo ilegítimo.

Dirección de los autores: Conservatoire et Jardin botaniques de la Ville de Genève, case postale 60, 1292 Chambésy, Suiza. E-mail: florapara@ville-ge.ch 


\section{Introducción}

En el curso de la digitalización de los tipos del Herbario $\mathrm{G}$, encontramos con regularidad nombres que necesitan una tipificación cuidadosa, de manera a determinar el material original utilizado por su autor. Con cierta frecuencia encontramos también errores de diferentes índoles que necesitan una corrección y de esta manera tener el tipo realmente designado por el autor. En la presente entrega, hemos tipificado todos los nombres del género Dorstenia L. publicados por E. Hassler y R. Chodat y cuyos tipos fueron colectados en Paraguay.

\section{Tipificaciones: Dorstenia L.}

1. Dorstenia brasiliensis f. balansae Chodat in Bull. Soc. Bot. Genève ser. 2, 11:257. 1920.

Holotypus: Paraguay. Guairá: "Doña Juana: près de Villa-Rica, dans les prairies", 17.IX.1874, Balansa, B. 1983 (G [G00381059]!). Isotypus: (G [G00381060]!, P).

El holotipo (Herbario Barbey-Boissier) lleva la anotación de la mano de R. Chodat: "Dorstenia brasiliensis Lam. Det. Chodat". El isotipo (Herbario de Candolle) no lleva ninguna anotación.

2. Dorstenia brasiliensis var. guaranitica Chodat in Bull. Soc. Bot. Genève ser. 2, 11:257. 1920 [nom. illeg.].

Lectotypus (designado por Berg, 2001 : 214): Paraguay. Cordillera: “Caacupé. II campm.”, 1914, Chodat, R. 247 (G [G00306982]!).

Syntypi: Paraguay. Cordillera: "Waldrand, sable, Rojas [CHODAT \& VISCHER, 1920: 258: "récoltée aux environs de San Bernardino, 247b»]", 1914, Chodat, R. $247 b$ (G [G00306981]!).

BrasiL. Estado de Bahia: "Bahia", s.f., Blanchet, J. S. 650 (G [G00381056]!). Estado de São Paulo: "St. Paul. Brésil”, s.f., Perdonnet, G. 182 (G [G00381055]!).

Chodat menciona en la descripción (CHODAT \& VISCHER, 1920: 258): "A cette forme [Dorstenia brasiliensis var. guaranitica Chodat] correspondent tous les numéros de Hassler: 3023, 927, 7847". De esta manera Chodat incluye en la var. guaranitica los tipos de tres nombres publicados anteriormente (véase abajo): Dorstenia brasiliensis var. major Chodat (1903, holotipo: Hassler 7847), Dorstenia brasiliensis var. palustris Hassl. (1919, holotipo: Hassler 3023), Dorstenia tubicina f. subexcentrica Hassl. (1919, holotipo: Hassler 927). Es entonces un nombre superfluo ilegítimo.

BERG (2001: 214) designa el lectotipo, pero el lugar de colección es indicado erróneamente ("San Bernardino") así como el colector ("Chodat \& Vischer"), corregimos aquí ambas indicaciones.
3. Dorstenia brasiliensis var. major Chodat in Bull. Herb. Boissier ser. 2, 3: 350. 1903.

Holotypus: ParaguaY. Amambay: "In campis in regione cursus superioris fluminis Apa", XI.1901-1902, Hassler, E. 7847 (G [G00381052]!). Isotypi: (G [G00306929]!, G [G00381014]!, G [G00381015]!, G [G00381051]!).

El holotipo (Herbario Chodat) lleva la anotación de la mano de R. Chodat: "Dorstenia brasiliensis Lam. var. major petiolis crassioribus foliis oblongis-ellipticis obtusis, 10/4 8/4 11/6". Los caracteres indicados por Chodat fueron utilizados textualmente en la descripción original. Los isotipos no llevan anotaciones de Chodat.

4. Dorstenia brasiliensis var. palustris Hassl. in Annuaire Conserv. Jard. Bot. Genève 21: 119. 1919.

Holotypus: Paraguay. Cordillera: "In uliginosis pr. lacus Ypacarai”, VI.1898-1899, Hassler, E. 3023 (G [G0030 6978]!). Isotypi : (G [G00306953]!, G [G00306979]!, G [G00306980]!).

El holotipo (Herbario Hassler) lleva la anotación de la mano de E. Hassler: "Dorstenia brasiliensis Lam. var. palustris Hassler". Los isotipos no llevan anotaciones de Hassler.

5. Dorstenia cayapia subf. lobata Hassl. in Annuaire Conserv. Jard. Bot. Genève $21: 116.1919$.

Holotypus: ParaguaY. Concepción: "Prope Concepcion in silvis", X.1901-1902, Hassler, E. 7598 a (G [G00381 046]!). Isotypi: (G [G00307705]!, G [G00381045]!).

Hassler describe este nombre caracterizado por hojas lobadas ("Foliis plus minus profunde 3-5 lobatis[...]"), indicando como holotipo "Hassler 7598 p.p. [pro parte]". Sin embargo, ninguno de los especímenes de la colección Hassler 7598 tiene hojas lobadas. Esta última colección es también un sintipo de la f. paraguariensis, sin indicación pro parte en la publicación original. La subf. lobata está incluída en la f. paraguariensis (HASSLER, 1919: 116).

Entre los demás sintipos de la f. paraguariensis, HASSLER (1919: 116) menciona una sola otra colección colectada por él: "Hassler 7598 a p.p.". En la colección Hassler 7598a, se encuentran tanto especímenes de hojas lobadas como de hojas no lobadas. Hemos conservado en la colección Hassler 7598 a todos los especímenes de hojas lobadas (G [G00307705], G [G00381045], G [G00381046]) y agrupado los especímenes de hojas no lobadas en la colección Hassler 7598a [bis] (G [G00381010], G [G00381047], G [G00381049], G [G003810 50]). Esta última colección corresponde al sintipo indicado por el autor de la f. paraguariensis. 
Al no haber especímenes de hojas lobadas en la colección Hassler 7598, designada como holotipo, pero sí en la colección Hassler 7598a, concluimos que se trata de un error tipográfico que corregimos aquí: se debe leer en la descripción original Hassler $7598 \underline{a}$ p.p. como tipo de de la subf. lobata, en lugar de Hassler 7598 p.p. El material visto por Hassler es el de su propia colección, puesto que el material conservado en aquel entonces en la Universidad de Ginebra (Herbario Chodat y Herbario Barbey-Boissier) no era accesible. La indicación del tipo citado por BERG $(2001: 211)$ debe también corregirse.

6. Dorstenia cayapia f. paraguariensis Hassl. in Annuaire Conserv. Jard. Bot. Genève 21: 116. 1919.

Lectotypus (colección designada por CARAUTA, 1978: 256; herbario precisado por BERG, 2001: 211; specimen in hoco loco designatum): Paraguay. Concepción: "N. Paraguay: zwischen Rio Apa und Rio Aquidaban", 1908-1909, Fiebrig, K. 4296 (G [G00174078]!). Isolectotypi: (BM, G [G00174077]!, GH, GOET, K, M, NY, P, US, Z).

Syntypi: ParaguaY. Concepción: "Nördl. Paraguay (22$23^{\circ}$ lat.) zwischen Rio Apa u. Aquidaban. San Luis", 19081909, Fiebrig, K. 4156 (G [G00174076]!, G [G0038 1009]!); "Prope Concepcion in silvis", X.1901-1902, Hassler, E. 7598 (BM, G [G00307704]!, G [G00381001]!, G [G00381003]!, G [G00381004]!, G [G00381048]!, GH, K, MPU, NY, P); "Prope Concepcion”, X.1901-1902, Hassler, E. 7598a[bis] (G [G00381010]!, G [G00381047]!, G [G00381049]!, G [G00381050]!).

CARAUTA (1978: 256) efectúa la lectotipificación de este nombre designando la colección Fiebrig 4296 en K; indica que no ha podido revisar todo el material de $\mathrm{G}$ al no encontrarse la colección de Fiebrig entre el material prestado y no excluye la posibilidad de designar ulteriormente otro mejor ejemplar si existiera en G. Cabe notar que Carauta cita el lectotipo como "Fiebrig 4296-4156", debido a la forma peculiar de la etiqueta de la colección Fiebrig: la segunda cifra "4156" es la mención de otra colección de Fiebrig indicando que ambas se parecen o son iguales.

BERG (2001: 211) indica que el lectotipo está en G, precisando de esta manera el herbario que conserva la colección original. Completamos aquí la lectotipificación de Carauta y Berg designando un único espécimen de la colección Fiebrig 4296 del Herbario Hassler (G [G00174078]), el ejemplar original conservado en G. El ejemplar del Herbario Delessert ( $\mathrm{G}$ [G00174077]) es un mero duplicado.

El sintipo Fiebrig 4156 está citado por HASSLER (1919: 116,120 ) bajo tres nombres diferentes como "Fiebrig 4156 p.p.". Cada uno corresponde a un espécimen distinto:
- "D. cayapia f. paraguariensis Hassler", una forma incluída en "D. cayapia var. typica Hassler" (HAssLER, 1919: 115). El ejemplar Fiebrig 4156 (G [G0038 1009]) del Herbario Hassler está anotado "Dorstenia cayapia Vell. var. typica Hassler" lo cual permite afirmar que se trata efectivamente de la colección original del sintipo mencionado por Hassler bajo su f. paraguariensis; el espécimen (G [G00174076]) del Herbario Delessert es un mero duplicado.

- “D. tubicina f. typica Hassler" (HASsLer, 1919: 120). El ejemplar del Herbario Hassler, sin etiqueta y con la sola indicación " 4156 ", corresponde al material que Hassler incluye en D. tubicina, encontrándose en el una mezcla de material que corresponde tanto a su $\mathrm{f}$. "typica" como a su f. "mayor". Hemos conservado en el ejemplar Fiebrig 4156[bis] (G [G00381011]) el material que corresponde a la f. "typica" (hojas de tamaño menor).

- "D. tubicina f. major (Chod.) Hassler" (Hassler, 1919: 120): las partes correspondientes a esta forma (hojas de tamaño mayor) han sido separadas del espécimen Fiebrig 4156[bis] y se encuentran agrupadas en el espécimen Fiebrig 4156[ter] (G [G00307907]).

En la etiqueta original del sintipo Fiebrig 4156 (G [G0038 1009]) de la f. paraguariensis están indicadas dos colecciones: "Fiebrig 4156 \& a", vale decir Fiebrig 4156 (G [G0017 4076], G [G00381009]) y Fiebrig $4156 a$ (G [G0038 1044]). La colecta Fiebrig $4156 a$ fue separada inicialmente por Hassler quién agregó una etiqueta independiente.

Los dos sintipos Hassler 7598 y el material agrupado en la colección Hassler 7598a [bis] no presentan hojas lobadas. De esta manera se distinguen del material típico de la subf. lobata incluída en la presente forma.

7. Dorstenia tubicina f. subexcentrica Hassl. in Annuaire Conserv. Jard. Bot. Genève 21: 119. 1919.

Holotypus: Paraguay. Cordillera: "In uliginosis pr. lag. Ypacarai”, IX.1885-1895, Hassler, E. 927 (G [G0030 6976]!). Isotypi : (BM, G [G00306963]!, G [G00306977]!, $\mathrm{K}, \mathrm{NY}, \mathrm{P})$.

HASSLER (1919: 119) menciona como tipo de este nombre la colección "Hassler 927 p.p.". Indica además que se trata de la colección determinada como " $D$. brasiliensis Chod. $P l$. Hassl. I, p. 59 non Lam." (G [G00306963], Herbario Chodat). En el momento de publicar su trabajo, el Herbario Chodat conservado en la Universidad de Ginebra no estaba disponible para Hassler y por lo tanto estas colecciones no fueron ni vistas ni anotadas por él. Existe un único ejemplar (Herbario Hassler) anotado de la mano de Hassler: "Dorstenia tubicina R. et P. var. opifera (Mart.) nob. f. subexcentrica Hassler no. 927 
det. E. Hassler". Este espécimen es el holotipo. Cabe observar que entre el material que forma el holotipo, se encuentra una hoja montada separadamente con la indicación "excentrica". Se trata probablemente de material perteneciente a D. excentrica Moric. visto por Hassler en el Herbario de Candolle (HASSLER, 1919: 119) que debe excluirse del material típico.

HASSLER (1919: 119) menciona bajo su "D. tubicina var. opifera" otra parte de la colección "Hassler 927 p.p.". Para poder separar claramente el material de la var. opifera y de la f. subexcentrica, hemos agrupado bajo la colección Hassler 927[bis] el material siguiente:

- Sobre la etiqueta del holotipo de la f. subexcentrica, arriba a la derecha, se encuentra una anotación "var. opifera nob Typica". Hemos separado la planta que corresponde a este lugar que va acompañada por un determinavit de la mano de Hassler "Dorstenia tubicina R. et P. var. opifera Mart.” (G [G00306952]).

- En el Herbario Hassler, se encuentra un ejemplar sin etiqueta y con la sola indicación "927" que corresponde a la descripción de Hassler en su "var. opifera" (G [G00306950]).

\section{Referencias}

BERG, C. C. (2001). Moreae, Artocarpeae, and Dorstenia (Moraceae). Fl. Neotropica 83.

Carauta, J. P. P. (1978). Dorstenia L. (Moraceae). Notas complementares II. Bradea 2 : 255-258.

Chodat, R. \& W. Vischer (1920). La végétation du Paraguay; IX. Urticiflores. Bull. Soc. Bot. Genève ser. 2, 11 : 226-258.

HASsler, E. (1919). Moracearum Paraguariensium conspectus. Annuaire Conserv. Jard. Bot. Genève 21 : 109-131. 